Aus dem Königlichen Krankenstift in Zwickau.

\title{
Ueber die Ortsbestimmung von Geschossen im Schädel.')
}

\author{
Von Prof. Dr. H. Braun.
}

Es ist mir kürzlich gelungen, einen in das Gehirn eingedrungenen Fremdkörper glücklich zu entfernen, und ich möchte das bei dieser Gelegenheit von uns ausgebildete Verfahren beschreiben, um diejenige Stelle der Schädeloberfläche zu bestimmen, von der ein auf Röntgenogrammen sichtbares Geschoß auf kürzestem Wege $\mathrm{zu}$ erreichen ist.

Es handelte sich um einen 131/2 jährioen Knaben, welcher am 22. August 1908 bewußtlos in seinem Zimmer gefunden wurde. Neben ihm lag ein abgeschossenes Tesching, über dem rechten Auge befand sich eine Einschußöffnung. Der zugezogene Arzt erweiterte die Einschußöffnung durch eine quere Inzision, stellte fest, da $\beta$ das Geschoß das Stirnbein oberhalb der Stirnhöhle durchdrungen hatte, und beschränkte sich sachgemäß auf Tamponade der Wunde. Der Verlauf war anfangs ein günstiger. Der Knabe wachte sehr bald auf und zeigte keinerlei allgemeine Hirnsymptome oder Herderscheinungen. Nur Kopfschmerzen stellten sich in den folgenden Tagen in allmählich zunehmender Intensität ein. Erst am 28. August zeigten sich eine Lähmung des linken Armes sowie Muskelzuckungren im Gebiet des rechten Facialis. Das Bewußtsein war anfangs stets ungetrübt, erst am 2. September stellte sich zeitweilig leichte Btnommenheit ein. Der Puls war anfangs ganz normal, niemals verlangsamt, am 28. August und in den folgenden 'Tagen betrug er 70-80 und wurde zeitweilig irregulär und flatternd. Die Temperatur, anfangs ebenfalls normal, stieg am 28. August auf 37,7, am 1. September auf 38. Die an der Einschußöffnung gesetzte Inzision fing zu eitern an, und bereits am 26. August zeigte sich hier ein Hirnprolaps, welcher allmählich die Wunde bis zum Hautniveau ausfüllte.

Am 3. September erfolgte die Ueberführung in das Königliche Krankenstift. Der hier erhobene Bef und ergibt sich gröBtenteils schon aus der vorstehenden Darstellung. Der Knabe war zeitweilig benommen, der rechte Arm war gelähmt. Die über dem rechten Auge befindliche Inzisionswunde sezernierte stark und war durch einen kleinen Hirnprolaps ausgefüllt. Die Temperatur betrug 37,3, der Puls 95. Der Knabe klagte andauernd über heftige Kopfschmerzen. In den folgenden Tagen nahm die Benommenheit zu, der Puls stieg am 6. September auf 100-115 und wurde flatternd. Ueber den Vorgang, durch den er verunglückt war, hatte der Kranke weder früher noch jetzt Auskunft geben können.

Es lag nunmehr die dringliche Indikation zur Entfernung des Fremdkörpers vor, da anzunehmen war, daß er nicht einheilen würde, sondern vielmehr bereits entzündliche Vorgänge oder eine intrakranielle Eiterung verursacht haben mubte. Die Symptome, welche der. Kranke zeigte, konnten ja nicht durch das Geschoß an sich, sondern nur darch sekundäre Prozesse bedingt sein. Die pathognomonische Bedeutung eines aus einer kleincn Schädelöffnung austretenden Hirnprolapses zeigte sich auf das eklatanteste. Es war dies hier das erste und mehrere Zwickau gehaltenen Vortrage. 
Tage einzige Symptom, welches auf den Eintritt ernster Komplikationen hinwies.

Die unterdessen aufgegommenen Röntgenogramme ließen einen etwa $21 / 2 \mathrm{~cm}$ langen, stabartigen Fremdkörper mit einer knopfartigen Verdickung an einem Ende erkennen. Ueber die Natur dieses Fremdkörpers ergingen wir uns in den kühnsten Vermutungen. Da an dem zum Tesching gehörigen Ladestock die Spitze abgebrochen und uns mitgeteilt worden war, daß der Ladestock ein verdicktes Ende gehabt habe, so hielten wir es für das Wahrscheinlichste, dab der Knabe beim Benutzen des Ladestocks dessen Ende abgebrochen und versucht hatte, letzteres mit Hilfe einer Patrone aus dem Lauf herauszubefördern. Wir sind da auf einer ganz falschen Fährte gewesen.

Der Fremdkörper mußte vom Einschuß über dem rechten Auge ziemlich genau von vorn nach hinten das Gehirn durchbohrt haben und mufte dicht unter dem rechten Scheitelbein liegen. Wir bestimmten als Stelle, wo wir zu seiner Entfernung einzugehen hatten, einen Punkt, welcher von der Nasenwurzel $17 \mathrm{~cm}$, in der Sagittallinie nach hinten gemessen, und zugleich $4,2 \mathrm{~cm}$ nach rechts von der Sagittallinie entfernt war. Wie wir die Lage dieses Punktes berechnet haben, werde ich später beschreiben. Mit der Lähmung des linken Armes konnten wir nichts anfangen, da der genannte Punkt erheblich über und hinter der Stelle gelegen war, wo die Rindenzentren für den linken Arm liegen mußten.

Die Operation wurde am 7. September in Aether-Chloroformnarkose ausgeführt. Das Operationsfeld wurde zunächst von einer Anzahl Einstichpunkten aus subkutan mit 0,5\% Novokainlösung mit Suprareninzusatz umspritzt, nicht um die Operation in Lokalanästhesie auszuführen, dazu war der Knabe viel zu unruhig, sondern um die Blutung zu verringern. Diese Umspritzung des Operationsfëldes, welche ich bei Schädeloperationen niemals unterlasse, macht alle älteren und neueren Verfahren zur provisorischen Blutstillung bei Schädeloperationen überflüssig. Bei ruhigen Kranken braucht man natürlich überhaupt kein Narcoticum.

Es wurde nun ein Haut-Knochenlappen mit unterer Basis umschnitten, dessen Mittelpunkt der oben erwähnte Punkt bildete, wo das Geschoß liegen mußte. Der Durchmesser der mit dem Borchardtschen Pflug umschnittenen Knochenscheibe betrug $5 \mathrm{~cm}$. Nach Aufheben des Lappens fand sich genau an der berechneten Stelle, in der Mitte des gesetzten Defekts eine Messingschraube von der beigezeichneten Form und Größe (Fig. 1). Der Gewindeteil der Schraube steckte noch in dem Loch, welches er in die Dura mater gerissen hatte, lag also halb intradural, während die andere Hälfte mit dem Schraubenkopf zwischen Dura und Schädel sich befand. An letzterer Stelle hatte sich ein kleiner Abszeß ge-

bildet, welcher die Dura vom Schädel abhob. Nach dem Herausziehen der Schraube aus der in der Dura befindlichen Oeffnung quoll aus dem non zugänglich gewordenen, nach vorn verlaufenden Schußkanal ein bräunlicher, aus Hirnbröckeln, Blut und Eiter bestehender Brei unter starkem Druck hervor. Mit einem feinen, scharfen Löffel wurde dieser Vorgang vorsichtig nnterstützt, ohne daß die Dura weiter eröffnet worden wäre. Die ausgeschnittene Knochenplatte mußte der Eiterung wegen entfernt werden. In den Schußkanal wurde ein langes, dünnes Drain eingeführt, das nebst einem Tampon am Rand des im übrigen durch Nähte in seiner Lage fixierten Hautlappens herausgeleitet wurde.

Die Operation wurde gut überstanden. Dann aber war der Zustand des Kranken eine Zeitlang ein sehr bedrohlicher. Heftige Kopfschmurzen, Nackenschmerzen, leichte Nackenstarre und hohe Temperaturen ließen den Eintritt einer septischen Meningitis befürchten. Jedoch vom 23. September ab trat allmähliche Besserung ein. Die Temperatur ging herunter und wurde am 30. September normal. Anfang Oktober war der körperliche Zustand gut geworden, das Sensorium war frei, die Lähmung des linken Armes war verschwunden. Psychisch bestand noch große Aufregung, Vergeßlichkeit und Demenz. Der Hirnprolaps an der Einschußöfnung hatte sich spontan abgestoßen. Der Hautlappen war angeheilt, jedoch hatte sich da, wo Drain und Tampon herausgeleitet waren, ebenfalls ein haselnußgroßer, pilzförmiger Hirnprolaps eingestellt. Letzterer wurde Ende Oktober mit der Schere abgetragen, worauf sich der Rest der Operationswunde in wenigen Tagen schloß. Zu gleicher Zeit war auch das psychische Verhalten des Knaben wiedèr ein völlig normales geworden. Intelligenz und $\mathrm{Ge}^{-}$ mütszustand waren die gleichen wie vor der Verletzung. Am 12. November, elf Wochen nach der Verletzung, wurde er geheilt entlassen. Er erinnerte sich nun auch, daß er die Messingschraube in einer Patrone, aus der die Kugel entfernt war, befestigt und in das Tesching gesteckt hatte.

Um die Stelle der Schädeloberfläche zu bestimmen, welcher das Geschob am nächsten liegen mußte, haben wir folgenden Weg eingeschlagen.

Es wurde bei einem Abstand der Antikathode von der Platte von $75 \mathrm{~cm}$ eine Röntgenaufnahme des Kopfes genal von vorn nach hinten gemacht und von dem gewonnenen Bild eine etwas erganzte Zeichnung hergestellt, welche die genauen Umrisse des Schattens der äußeren Haut, der Schädelknochen und des Geschosses enthielt.

Fig. 2 zeigt diese Zeichnung verkleinert. $S$ ist die konstruierte Sagittalebene, A-B der größte quere Durchmesser. Aus

Fig. 2.

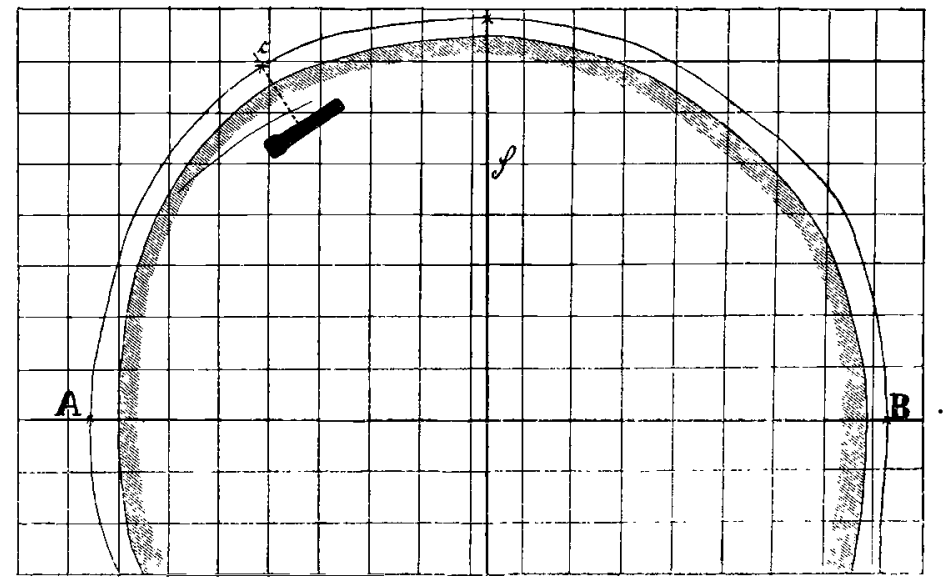

dem Bild ergibt sich, daß der gesuchte Punkt $\mathrm{c}$ in einer Linie gelegen sein muß, welche in einer bestimmten Entfernung rechts (auf der Zeichnung links) von der Sagittallinie, dieser parallel auf der Schädeloberfläche gezogen wird. Es handelt sich nur darum, die Entfernung c-d zu messen. Um hierzu ein objektives Maß zu gewinnen, ist es nötig, das infolge der Divergenz der Strahlen vergrößerte Röntgenbild auf natürliche Größe zu reduzieren. Das geschieht in folgender Weise. Ich messe anf der Zeichnung 2 die Entfernung A-B, also den gröBten queren Durchmesser, und finde sie $20,5 \mathrm{~cm}$ lang. Ich messe ferner mit dem Tasterzirkel den wirklichen größten queren Durchmesser am Kopf des Kranken und finde ihn $15 \mathrm{~cm}$ lang. Ich $\mathrm{muB}$ also, um auf der Zeichnung objektive Maße ablesen zu können, eine von 1,3 auf 1 reduzierte Zeich nung herstellen, deren orößter querer Durchmesser dann $15 \mathrm{~cm}$, wie in Wirklichkeit, lang ist. Die Ausführung dieser Reduktion erfolgt in der bekannten einfachen Weise. Man teilt die Fig. ? in Quadrate mit einer Seitenlänge von $1,3 \mathrm{~cm}$ ein und ïberträgt die Linien der Zeichrung auf ein Papier, welches in Quadrate mit einer Seitenlänge von $1 \mathrm{~cm}$ geteilt ist. Fig. 3 zeigt die reduzierte Zeich-

Fig. 3.

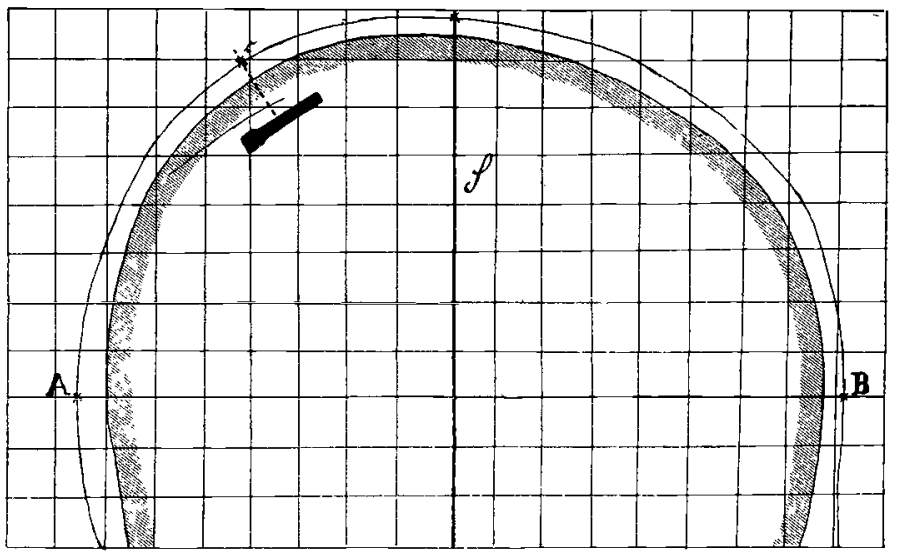

nung verkleinert.1) An ihr kann ich die Entfernung $\mathrm{c} d$ mit $4,2 \mathrm{~cm}$ messen. Die Stelle, wo das GeschoB aufzusuchen ist, liegt also in einer zur Sagittallinie parallelen, auf der Schädeloberfläche gezogenen Linie, welche rechts von der Sagittallinie und $4,2 \mathrm{~cm}$ von ihr entfernt gelegen ist. In derselben Weise wurde nun bei

1) A nmerkung bei der Korrektur: Leider ist die Reproduktion der beiden Zeichnungen 2 und 3 nicht in gleichein MaBstabe der Verkleinerung erfolgt. 
gleichem Röhrenabstand eine Röntgenaufnahme des Kopfes genau von links nach rechts hergestellt, darnach wieder eine Umrißzeichnung angefertigt und diese von 1,3 auf 1 , zur natürlichen Größe, reduziert.

Es genügt, diese reduzierte Zeichnung in Fig. 4 verkleinert wiederzugeben. Es markiert sich auf ihr bei a sehr scharf die

Fig. 4.

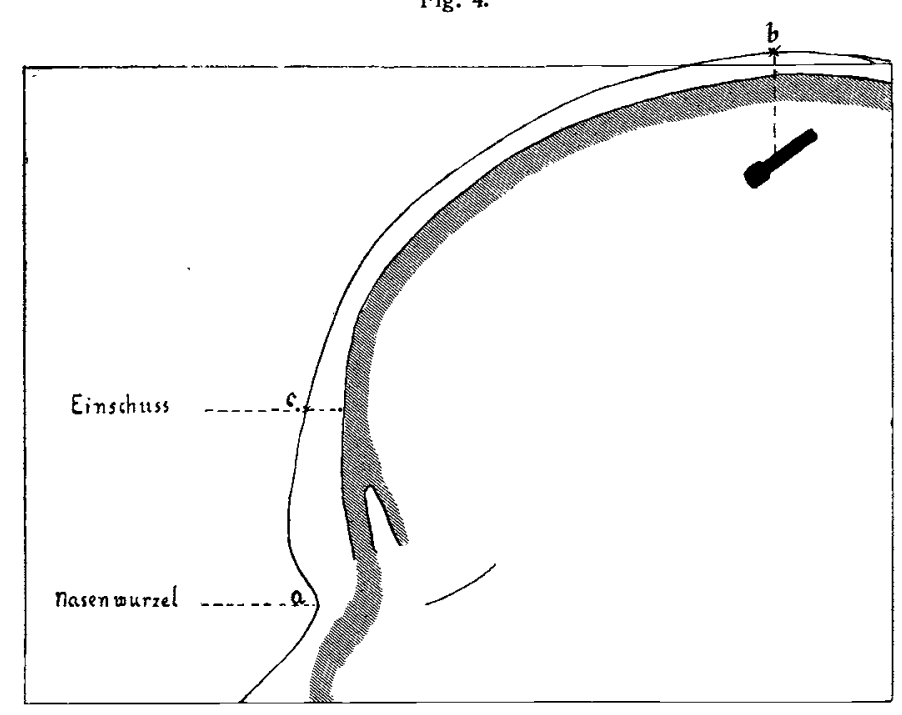

Nasenwurzel, auch ist bei c im Photogramm der Einschuß zu erkennen. Ich messe nun, natürlich der Kurve der Hautoberfläche folgend, die Entfernung a b. Sie beträgt $17 \mathrm{~cm}$. Der Punkt, wo das Geschoß zu finden ist, muß also in einer auf der Schädeloberfläche gezogenen queren Linie liegen, welche in der Sagittallinie $17 \mathrm{~cm}$ von der Nasenwurzel entfernt ist und einen rechten Winkel mit der Sagittallinie bildet.

Die beiden gefundenen Linien wurden nunmehr auf der Kopfoberfläche des Kranken aufgetragen, in ihrem Schnittpunkt, also $17 \mathrm{~cm}$ hinter der Nasenwurzel und $4,2 \mathrm{~cm}$ rechts von der Sagittallinie, mußte eingegangen werden, um zu dem Geschoß zu gelangen. Es ist bereits gesagt worden, daß letzteres genau an der berechneten Stelle gefunden wurde.

Es ist klar, daß diè vorstehende Konstruktion nicht im mathematischen Sinn exakt sein kann, namentlich deshalb nicht, weil in einem Röntgenbild des Kopfes nicht genau dessen größter Durchmesser abgebildet wird, sondern infolge der Divergenz der Röntgenstrahlen, die von der Antikathode ausgehen, ein kleinerer, der Röntgenröhre näher gelegener Durchmesser. Es wird dies sofort durch Betrachtung der Fig. 5 verständlich, wo der Schädel durch eine Kugel ange-

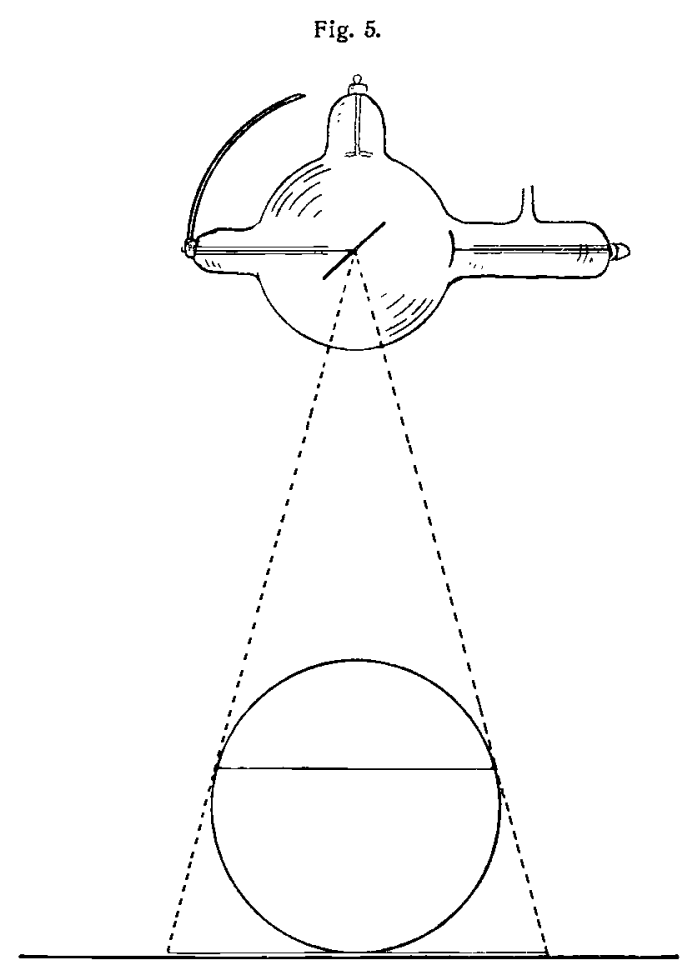

deutet ist. Man sieht, daß diejenigen Strahlen, welche den Schatten der Konturen der Schädeloberfläche geben müssen, die Kugel nicht an deren Aequator treffen. Die Berechnung der natürlichen Größe des Röntgenbildes aus dem Vergleich der Länge des abgebildeten und des wirklichen größten queren Durchmessers des Kopfes kann daher nicht mathematisch richtig sein. Eine weitere Fehlerquelle liegt darin, daf das Röntgenbild nicht, wie angenommen, ein einfach vergröfertes, sondern in Wirklichkeit auch verzeichnetes Schattenbild eines Gegenstandes ist.

Ich glaube indessen, daf diese Fehler der praktischen Brauchbarkeit des Verfahrens keinen Eintrag tun werden. Für die Praxis reicht seine Genauigkeit aus, wenigstens wenn es sich um Fremdkörper im Schädel handelt.

Möglich ist, daß die Fehler zu groß werden, wenn es sich um Fremdkörper handelt, welche in Körperteilen mit viel größeren Dimensionen, wie im Brustkorb, gelegen sind.

Ueber die Entfernung des Fremdkörpers von der Schädeloberfläche, also über die Tiefe, in welcher er sich befindet, gibt unser Verfahren keinen Aufschluß. Dasselbe beschränkt sich vielmehr darauf, die Stelle der Schädeloberfläche zu bestimmen, welche dem Flemdkörper am nächsten gelegen ist. Letzteres dürfte wohl auch für die Praxis das Wichtigste sein. Einen annähernden und genügend orientierenden Anhalt darüber, wie tief ein Geschoß im Gehirn sitzt, wie weit es ungefähr von der Stelle entfernt ist, die man zum Operationsfeld bestimmt hat, gewinnt man ja natürlich stets ohne weiteres, wenn man zwei in rechtwinklig zueinander gestellten Ebenen aufgenommene Röntgenbilder betrachtet, auf denen der Fremdkörper zu sehen ist. Daß in unserem Fall das Geschoß ziemlich dicht unter dem rechten Scheitelbein liegen mußte, hatten uns die Röntgenaufnahmen schon vor der Konstruktion der Zeichnungen gesagt. 